\title{
A collocation method for linear integral equations in terms of the generalized Bernstein polynomials
}

\author{
Aysegul Akyuz-Dascioglu ${ }^{1}$ and Nese Isler Acar ${ }^{2}$ \\ ${ }^{1}$ Department of Mathematics, Faculty of Arts \& Sciences, Pamukkale University, Denizli, Turkey \\ ${ }^{2}$ Department of Mathematics, Faculty of Arts \& Sciences, Mehmet Akif Ersoy University, Burdur, Turkey
}

Received: 21 August 2015, Revised: 12 September 2015, Accepted: 15 September 2015

Published online: 10 February 2016.

\begin{abstract}
In this study, a collocation method based on the generalized Bernstein polynomials is presented and analized for the solution of linear Fredholm-Volterra integral equations (FVIEs). Error bounds and convergence of this method are demonstrated. Some examples are also given to illustrate the accuracy, efficiency and applicability of the method.
\end{abstract}

Keywords: Bernstein polynomial approach, linear integral equations, collocation method.

\section{Introduction}

Integral equations have been first produced in connection with the famous tautochrone problem by Abel (Italian mathematician) in 1825. These equations are defined as equations in which the unknown function appears under the integral sign. Besides, the most frequently used integral equations are collected two major classes, namely Volterra and Fredholm integral equations. Integral equations are closely related both in pure and applied mathematics. These equations play an important role in various fields of science and numerous applications such as elasticity, plasticity, heat and mass transfer, oscillation theory, fluid dynamics, filtration theory, electrostatics, electrodynamics, biomechanics, game theory, control, queuing theory, electrical engineering, economics, medicine, etc. Many problems included ordinary or partial differential equations can be converted to problems of the integral equations. Therefore, many researchers are interested in numerical methods to get the solution of integral equations.

Bernstein polynomials and their basis polynomials $[5,6]$ defined on the interval $[0,1]$, can be easily generalized to the interval $[a, b]$ by using transformation $t=\frac{x-a}{b-a}$ as follows.

Definition 1. Generalized Bernstein basis polynomials can be defined on the interval $[a, b]$; by

$$
p_{i, n}(x)=\frac{1}{(b-a)^{n}}\left(\begin{array}{c}
n \\
i
\end{array}\right)(x-a)^{i}(b-x)^{n-i} ; i=0,1, \ldots, n \text {. }
$$

Definition 2. Let y be continuous function on the interval $[a, b]$. Generalized Bernstein polynomials of nth-degree are defined by

$$
B_{n}(y ; x)=\sum_{i=0}^{n} y\left(a+\frac{(b-a) i}{n}\right) p_{i, n}(x)
$$

such that $p_{i, n}(x)$ are the generalized Bernstein basis polynomials. 
The generalized Bernstein polynomials and their basis forms have many useful properties, such as the positivity, continuity, recursion's relation, symmetry, unity partition of the basis set over the interval $[a, b]$, uniform approximation, differentiability and integrability. For this reason, these polynomials can be useful mathematical tools for numerical analysis and approximation theory. In recent years, these polynomials are ecountered in research works of numerical solution of Volterra $[2,7,12]$ and Fredholm $[8,11]$ integral equations.

Theorem 1. If $y(x)$ is continuous function on the interval $[a, b]$, then

$$
\lim _{n \rightarrow \infty} B_{n}(y ; x)=y(x)
$$

converges uniformly.

Proof. Proof of the above theorem can be easily seen from the proof of theorem given on the interval $[0,1]$ by Phillips $[9]$.

Definition 3. Linear Fredholm -Volterra integral equation of the third kind is given by:

$$
a(x) y(x)=g(x)+\lambda_{1} \int_{a}^{b} f(x, t) y(t) d t+\lambda_{2} \int_{a}^{x} v(x, t) y(t) d t
$$

such that $a(x) \neq 0$ and $a(x) \neq 1$. Here $a(x), g(x) \in[a, b], f(x, t):[a, b] \times[a, b] \rightarrow \mathbb{R}$ and $v(x, t):[a, b] \times[a, b] \rightarrow \mathbb{R}$ are continuous functions, $\lambda_{1}$ and $\lambda_{2}$ are constants, $y(x)$ is unknown function. Privately the equation (1) is called linear Fredholm-Volterra integral equations of the first and second kind respectively for $a(x) \equiv 0$ and $a(x) \equiv 1$.

The reminder of this paper consists four sections: In Section 2, a collocation method is developed directly to get the numerical solution of the integral equations by means of the generalized Bernstein polynomials. In Section 3, error bounds and convergence analysis are given for the proposed method. Section 4 demonstrates the applicability of the presented method. In this part, some linear examples are solved and compared with different methods. Finally, Section 5 is ended with conclusions contained general inferences of the study.

\section{Method of solution}

The aim of the proposed method is to derive a collocation method for approximate solution of the linear FVIE (1) by means of the generalized Bernstein polynomials:

$$
y(x) \cong B_{n}(y ; x)=\sum_{i=0}^{n} y\left(a+\frac{(b-a) i}{n}\right) p_{i, n}(x) .
$$

Now, we give a theorem that represents a main matrix relation for the linear integral equation (1).

Theorem 2. Let $x_{s}$ be collocation points and $y(x)$ be continuous function on the interval $[a, b]$. The linear FVIE (1) has the following matrix form:

$$
\left[\mathbf{A P}-\lambda_{1} \mathbf{F}-\lambda_{2} \mathbf{V}\right] \mathbf{Y}=\mathbf{G}
$$

Here $\mathbf{A}=\operatorname{diag}\left[a\left(x_{s}\right)\right], \mathbf{F}=\left[F_{s, i}\right], \mathbf{V}=\left[V_{s, i}\right], \mathbf{P}=\left[p_{i, n}\left(x_{s}\right)\right]$ are $(n+1) \times(n+1)$ matrices, and $\mathbf{Y}=\left[y\left(a+\frac{(b-a) i}{n}\right)\right]$, $\mathbf{G}=\left[g\left(x_{s}\right)\right]$ are $(n+1) \times 1$ matrices for $i, s=0,1, \ldots, n$. 
Proof. Since solution of the equation (1) is continuous function on the interval $[a, b]$, it has the Bernstein polynomial approach given with the expression (2) from the Theorem 1. So the solution function can be written as the matrix form:

$$
y(x) \simeq \mathbf{P}(x) \mathbf{Y}
$$

such that

$$
\mathbf{P}(x)=\left[p_{0, n}(x) p_{1, n}(x) \ldots p_{n, n}(x)\right], \quad \mathbf{Y}=\left[y(a) y\left(a+\frac{b-a}{n}\right) \ldots y(b)\right]^{T}
$$

Substituting the collocation points and the relation (4) into the equation (1), we obtain a linear algebraic equation system

$$
a\left(x_{s}\right) \mathbf{P}\left(x_{s}\right) \mathbf{Y}=g\left(x_{s}\right)+\lambda_{1} \int_{a}^{b} f\left(x_{s}, t\right) \mathbf{P}(t) d t \mathbf{Y}+\lambda_{2} \int_{a}^{x_{s}} v\left(x_{s}, t\right) \mathbf{P}(t) d t \mathbf{Y}
$$

Here $y\left(x_{s}\right)=B_{n}\left(y ; x_{s}\right)(s=0,1, \ldots, n)$ because of the collocation method. If the integrals at the sides of $\lambda_{1}$ and $\lambda_{2}$ are called respectively $\mathbf{F}\left(x_{s}\right)$ and $\mathbf{V}\left(x_{s}\right)$, then for $i=0,1, \ldots, n$, the elements of these matrices can be written as

$$
\begin{aligned}
& \mathbf{F}\left(x_{s}\right)=\left[\begin{array}{lllll}
F_{s, 0} & F_{s, 1} & \ldots & F_{s, n}
\end{array}\right] ; \quad F_{s, i}=\int_{a}^{b} f\left(x_{s}, t\right) p_{i, n}(t) d t \\
& V\left(x_{s}\right)=\left[\begin{array}{lllll}
V_{s, 0} & V_{s, 1} & \ldots & V_{s, n}
\end{array}\right] ; \quad V_{s, i}=\int_{a}^{x_{s}} v\left(x_{s}, t\right) p_{i, n}(t) d t .
\end{aligned}
$$

Therefore the equation (5) becomes

$$
\left[a\left(x_{s}\right) \mathbf{P}\left(x_{s}\right)-\lambda_{1} \mathbf{F}\left(x_{s}\right)-\lambda_{2} \mathbf{V}\left(x_{s}\right)\right] \mathbf{Y}=g\left(x_{s}\right)
$$

For $s=0,1, \ldots, n$, this system is written by the matrix equation (3), and this is completed the proof.

Corollary 1. The matrix equation (3) can be reduced the following matrix forms respectively for linear FVIEs of the first and second kind:

$$
\left[-\lambda_{1} \mathbf{F}-\lambda_{2} \mathbf{V}\right] \mathbf{Y}=\mathbf{G} \text { and }\left[\mathbf{P}-\lambda_{1} \mathbf{F}-\lambda_{2} \mathbf{V}\right] \mathbf{Y}=\mathbf{G}
$$

Now we follow the some steps to solve the linear FVIE (1).

(i) Let the equation (3) be written in the compact form

$$
\mathbf{W Y}=\mathbf{G} \quad \text { or }[\mathbf{W} ; \mathbf{G}]
$$

so that $\mathbf{W}=\mathbf{A P}-\lambda_{1} \mathbf{F}-\lambda_{2} \mathbf{V}$. The equation corresponds a linear algebric equation system with $n$ unkowns.

(ii) If $\operatorname{rank}(\mathbf{W})=\operatorname{rank}[\mathbf{W} ; \mathbf{G}]=n+1$, then solution of this system is uniquely determined. This kind of systems can be solved by the Gauss elimination, generalized inverse, LU and QR factorization methods. 


\section{Convergence and error analysis}

Definition 4. Error is denoted by $e_{n}(x)=y(x)-y_{n}(x)$ such that $y(x)$ is an exact solution and $y_{n}(x)=B_{n}(y ; x)$ is a generalized Bernstein approximate solution. Then maximum error can be defined as

$$
\left\|e_{n}\right\|_{\infty}=\max _{a \leq x \leq b}\left|e_{n}(x)\right|
$$

Let $y\left(x_{s}\right) \neq 0$ and $B_{n}\left(y ; x_{s}\right)$ be scalars. Then maximum, mean, root of the mean square and absolute relative errors at the collocation points can be numerically computed respectively by

$$
\begin{aligned}
& E_{\max }=\max _{x_{s} \in[a, b]}\left|e_{n}\left(x_{s}\right)\right|, \quad E_{\text {mean }}=\frac{1}{n+1} \sum_{s=0}^{n}\left|e_{n}\left(x_{s}\right)\right|, \\
& E_{\text {root }}=\sqrt{\frac{1}{n+1} \sum_{s=0}^{n}\left(e_{n}\left(x_{s}\right)\right)^{2}}, \quad E_{\text {rel }}=\frac{\left|e_{n}\left(x_{s}\right)\right|}{\left|y\left(x_{s}\right)\right|} .
\end{aligned}
$$

Besides, residual error of the presented method can be defined by

$$
R_{n}(x)=a(x) B_{n}(y ; x)-\lambda_{1} \int_{a}^{b} f(x, t) B_{n}(y ; t) d t-\lambda_{2} \int_{a}^{x} v(x, t) B_{n}(y ; t) d t-g(x)
$$

Definition 5. [10] Let $y(x)$ be defined on $[a, b]$, the modulus of contunity of $y(x)$ on $[a, b], \omega(\delta)$, is defined for $\delta>0$ by

$$
\omega(\delta)=\sup _{\substack{x_{1}, x_{2} \in[a, b] \\\left|x_{1}-x_{2}\right| \leq \delta}}\left|y\left(x_{1}\right)-y\left(x_{2}\right)\right|
$$

Lemma 1. $[10] y(x)$ is uniformly continuous on $[a, b]$ iff $\lim _{\delta \rightarrow 0} \omega(\delta)=0$.

Theorem 3. (Uniqueness Theorem) Let the function $a(x) \in \mathbb{C}[a, b]$ and the kernels $f, v \in \mathbb{C}\left([a, b]^{2}\right)$. The equation (1) has a unique solution whenever $0<q<1, q=M_{1}+\left|\lambda_{1}\right| M_{2}(b-a)+\left|\lambda_{2}\right| M_{3}(b-a)$.

Proof. Let $T: \mathbb{C}[a, b] \rightarrow \mathbb{C}[a, b]$ be a mapping. Then the equation (1) can be rewritten as

$$
\begin{gathered}
T y=a(x) y(x)-g(x)-\lambda_{1} \int_{a}^{b} f(x, t) y(t) d t-\lambda_{2} \int_{a}^{x} v(x, t) y(t) d t, \\
T y^{*}=a(x) y^{*}(x)-g(x)-\lambda_{1} \int_{a}^{b} f(x, t) y^{*}(t) d t-\lambda_{2} \int_{a}^{x} v(x, t) y^{*}(t) d t
\end{gathered}
$$

for $y, y^{*} \in \mathbb{C}[a, b]$ such that $y=T y$ and $y^{*}=T y^{*}$.

Besides, $a(x), f(x, t)$ and $v(x, t)$ are bounded functions such that $|a(x)| \leq M_{1},|f(x, t)| \leq M_{2}$ and $|v(x, t)| \leq M_{3}$; $M_{1}, M_{2}, M_{3}>0$ because of $a(x) \in \mathbb{C}[a, b]$ and $f, v \in \mathbb{C}\left([a, b]^{2}\right)$. Considering the maximum norm for one variable 
function, we have

$$
\begin{aligned}
\left\|T y-T y^{*}\right\|_{\infty} & \leq M_{1}\left\|y-y^{*}\right\|_{\infty}+\left|\lambda_{1}\right| M_{2}\left\|y-y^{*}\right\|_{\infty} \int_{a}^{b} d t+\left|\lambda_{2}\right| M_{3}\left\|y-y^{*}\right\|_{\infty} \int_{a}^{x} d t \\
& \leq M_{1}\left\|y-y^{*}\right\|_{\infty}+\left|\lambda_{1}\right| M_{2}(b-a)\left\|y-y^{*}\right\|_{\infty}+\left|\lambda_{2}\right| M_{3}(b-a)\left\|y-y^{*}\right\|_{\infty} \\
& \leq\left(M_{1}+\left|\lambda_{1}\right| M_{2}(b-a)+\left|\lambda_{2}\right| M_{3}(b-a)\right)\left\|y-y^{*}\right\|_{\infty} .
\end{aligned}
$$

Denoting $q=M_{1}+\left|\lambda_{1}\right| M_{2}(b-a)+\left|\lambda_{2}\right| M_{3}(b-a)$ such that $0<q<1$, the above inequality is

$$
\left\|T y-T y^{*}\right\|_{\infty} \leq q\left\|y-y^{*}\right\|_{\infty} .
$$

From well-known Banach fixed-point theorem, there exict a unique solution of the equation (1) and this completes the proof.

Theorem 4. [1] Let $B_{n}(y ; x)$ be generalized Bernstein polynomial solution on the interval $[a, b]$. If $y(x)$ is continuous function on the interval $[a, b]$, then the following inequality and uniform convergence are hold:

$$
\left|e_{n}(x)\right| \leq \omega\left(n^{-1 / 2}\right)(1+\sqrt{(x-a)(b-x)}) \text { and } \lim _{n \rightarrow \infty}\left\|e_{n}\right\|_{\infty}=0 .
$$

Here $\omega(\delta)$ is modulus of contunity of $y(x)$ on the interval $[a, b]$.

Theorem 5. Let $a(x), y(x)$ be continuous functions on the interval $[a, b]$ and $f(x, t), v(x, t)$ be continuous functions on the square $[a, b] \times[a, b]$. Then residual error of the generalized Bernstein polynomial approach holds for the linear FVIE (1) as follows:

$$
\left\|R_{n}\right\|_{\infty} \leq\left(\alpha+(b-a)\left|\lambda_{1}\right| \beta+(x-a)\left|\lambda_{2}\right| \gamma\right)\left\|e_{n}\right\|_{\infty} \text { and } \lim _{n \rightarrow \infty}\left\|R_{n}\right\|_{\infty}=0 .
$$

Here $\alpha=\|a\|_{\infty}, \beta=\|f\|_{\infty}$ and $\gamma=\|v\|_{\infty}$ are positive constants.

Proof. Since $y$ is continuous function on the interval $[a, b]$, it can be denoted as Bernstein approximate solution. Substituting $g(x)$ given in the equation (1) to the absolute value of the residual error (6), the following inequality can be written for the residual error:

$$
\begin{aligned}
\left|R_{n}(x)\right| & =\left|a(x) B_{n}(y ; x)-\lambda_{1} \int_{a}^{b} f(x, t) B_{n}(y ; t) d t-\lambda_{2} \int_{a}^{x} v(x, t) B_{n}(y ; t) d t-g(x)\right| \\
& \leq|a(x)|\left|B_{n}(y ; x)-y(x)\right|+\left|\lambda_{1}\right| \int_{a}^{b}|f(x, t)|\left|B_{n}(y ; t)-y(t)\right| d t+\left|\lambda_{2}\right| \int_{a}^{x}|v(x, t)|\left|B_{n}(y ; t)-y(t)\right| d t .
\end{aligned}
$$

Moreover, by using definitions of the maximum error and properties of the norm for one and two variables functions, the residual error bound is obtained as:

$$
\begin{aligned}
\left\|R_{n}\right\|_{\infty} & \leq\|a\|_{\infty}\left\|e_{n}\right\|_{\infty}+(b-a)\left|\lambda_{1}\right|\|f\|_{\infty}\left\|e_{n}\right\|_{\infty}+(x-a)\left|\lambda_{2}\right|\|v\|_{\infty}\left\|e_{n}\right\|_{\infty} \\
& \leq\left(\|a\|_{\infty}+(b-a)\left|\lambda_{1}\right|\|f\|_{\infty}+(x-a)\left|\lambda_{2}\right|\|v\|_{\infty}\right)\left\|e_{n}\right\|_{\infty} .
\end{aligned}
$$

Denoting $\alpha=\|a\|_{\infty}=\max _{x \in[a, b]}|a(x)|, \beta=\|f\|_{\infty}=\max _{x, t \in[a, b]}|f(x, t)|$ and $\gamma=\|v\|_{\infty}=\max _{x, t \in[a, b]}|v(x, t)|$, the desired inequality is founded. 
In view of the Theorem 4, the following relation

$$
\left\|R_{n}\right\|_{\infty} \leq\left(\alpha+(b-a)\left|\lambda_{1}\right| \beta+\max _{x \in[a, b]}(x-a)\left|\lambda_{2}\right| \gamma\right)\left\|e_{n}\right\|_{\infty} \leq c\left\|e_{n}\right\|_{\infty}
$$

and uniform convergence of the residual error are also proved. Here $c=\alpha+(b-a)\left|\lambda_{1}\right| \beta+(b-a)\left|\lambda_{2}\right| \gamma$ is positive constant.

Corollary 2. The residual error bounds of linear FVIEs of the first and second kind are respectively

$$
\begin{aligned}
& \left\|R_{n}\right\|_{\infty} \leq\left((b-a)\left|\lambda_{1}\right| \beta+(x-a)\left|\lambda_{2}\right| \gamma\right)\left\|e_{n}\right\|_{\infty}, \\
& \left\|R_{n}\right\|_{\infty} \leq\left(1+(b-a)\left|\lambda_{1}\right| \beta+(x-a)\left|\lambda_{2}\right| \gamma\right)\left\|e_{n}\right\|_{\infty} .
\end{aligned}
$$

Here $\alpha, \beta$ and $\gamma$ are positive constants denoted as the Theorem 5.

Theorem 6. Let $y$ be a continuous function and $x_{s}(s=0,1, \ldots, n)$ be collocation points on the interval $[a, b]$. Then, the residual error bound at the collocation points for linear FVIEs of the first, second and third kind are

$$
\left|R_{n}\left(x_{s}\right)\right|<\kappa\left(x_{s}\right)(b-a)\left(1+\frac{b-a}{2}\right) \omega\left(n^{-1 / 2}\right)
$$

and $\lim _{n \rightarrow \infty}\left|R_{n}\left(x_{s}\right)\right|=0$. Here $\kappa$ is a positive constant depended on the collocation points.

Proof. Since $y$ is continuous function on the interval $[a, b]$, it can be denoted as Bernstein approximate solution. So absolute value of residual error can be expressed at the collocation points for linear FVIE (1) of the third kind as

$$
\left|R_{n}\left(x_{s}\right)\right| \leq\left|a\left(x_{s}\right)\right|\left|B_{n}\left(y ; x_{s}\right)-y\left(x_{s}\right)\right|+\left|\lambda_{1}\right| \int_{a}^{b}\left|f\left(x_{s}, t\right)\right|\left|B_{n}(y ; t)-y(t)\right| d t+\left|\lambda_{2}\right| \int_{a}^{x_{s}}\left|v\left(x_{s}, t\right)\right|\left|B_{n}(y ; t)-y(t)\right| d t
$$

Since $B_{n}\left(y ; x_{s}\right)=y\left(x_{s}\right)(s=0,1, \ldots, n)$ from the collocation method and from the Theorem 4 , the following inequality can be written for the absolute residual error:

$$
\left|R_{n}\left(x_{s}\right)\right| \leq \omega\left(n^{-1 / 2}\right)\left\{\left|\lambda_{1}\right| \int_{a}^{b}\left|f\left(x_{s}, t\right)\right|[1+\sqrt{(t-a)(b-t)}] d t+\left|\lambda_{2}\right| \int_{a}^{x_{s}}\left|v\left(x_{s}, t\right)\right|[1+\sqrt{(t-a)(b-t)}] d t\right\}
$$

Denoting $\varepsilon_{s}=\varepsilon\left(x_{s}\right)=\left|\lambda_{1}\right| \max _{t \in[a, b]}\left|f\left(x_{s}, t\right)\right|, \rho_{s}=\rho\left(x_{s}\right)=\left|\lambda_{2}\right| \max _{t \in[a, b]}\left|v\left(x_{s}, t\right)\right|$, and taking $\max _{t \in[a, b]}[1+\sqrt{(t-a)(b-t)}]=1+\frac{b-a}{2}$ the residual error becomes

$$
\begin{aligned}
\left|R_{n}\left(x_{s}\right)\right| & \leq\left((b-a) \varepsilon_{s}+\left(x_{s}-a\right) \rho_{s}\right)\left(1+\frac{b-a}{2}\right) \omega\left(n^{-1 / 2}\right) \\
& \leq\left(\varepsilon_{s}+\rho_{s}\right)(b-a)\left(1+\frac{b-a}{2}\right) \omega\left(n^{-1 / 2}\right) \\
& \leq \kappa\left(x_{s}\right)(b-a)\left(1+\frac{b-a}{2}\right) \omega\left(n^{-1 / 2}\right) .
\end{aligned}
$$


Table 1: Mean errors of Example 1.

\begin{tabular}{|c|c|c|c|c|c|}
\hline$n$ & $x_{s}=\frac{s}{n}$ & $x_{s}=\cos \left(\frac{\pi s}{n}\right)$ & $n$ & $x_{s}=\frac{s}{n}$ & $x_{s}=\cos \left(\frac{\pi s}{n}\right)$ \\
\hline 2 & 0 & $3.7 e-017$ & 11 & $2.7 e-015$ & $3.3 e-016$ \\
\hline 3 & $4.9 e-017$ & $1.1 e-016$ & 12 & $1.4 e-015$ & $3.6 e-016$ \\
\hline 4 & $2.5 e-017$ & $1.8 e-016$ & 13 & $4.4 e-015$ & $2.5 e-016$ \\
\hline 5 & $3.6 e-016$ & $5.4 e-017$ & 14 & $2.6 e-015$ & $1.7 e-016$ \\
\hline 6 & $5.4 e-016$ & $9.8 e-017$ & 16 & $1.5 e-015$ & $3.5 e-016$ \\
\hline 7 & $4.2 e-016$ & $9.8 e-017$ & 17 & $2.1 e-014$ & $2.7 e-016$ \\
\hline 8 & $2.8 e-016$ & $2.4 e-016$ & 18 & $2.5 e-014$ & $2.2 e-016$ \\
\hline 9 & $1.2 e-016$ & $2.0 e-016$ & 19 & $8.8 e-014$ & $3.2 e-016$ \\
\hline 10 & $2.4 e-016$ & $2.0 e-016$ & 20 & $1.5 e-013$ & $4.0 e-016$ \\
\hline
\end{tabular}

Here $\kappa\left(x_{s}\right)$ is bigger constant than $\varepsilon_{s}$ and $\rho_{s}$ for $a \leq x_{s} \leq b$. Since $\omega\left(n^{-1 / 2}\right) \rightarrow 0$ in view of the Lemma $1,\left|R_{n}\left(x_{s}\right)\right| \rightarrow 0$ as $n \rightarrow \infty$. The proof is completed. Besides, this theorem is hold for linear FVIEs of the first and second kind because of $B_{n}\left(y ; x_{s}\right)=y\left(x_{s}\right)$.

\section{Numerical results}

Four numerical examples are given by using the Bernstein collocation method. The collocation points are selected as uniform $x_{s}=a+\frac{(b-a) s}{n}$ and nonuniform $x_{s}=\frac{(a+b)-(b-a) \cos \left(\frac{\pi s}{n}\right)}{2} ; s=0,1, \ldots, n$. Numerical results computed in MATLAB 7.1 with 32 digits are compared with the other methods and they are presented via the Tables and Figures.

Example 1. Consider the

$$
y(x)=1+\int_{-1}^{1}\left(x t+x^{2} t^{2}\right) y(t) d t ; \quad-1 \leq x \leq 1
$$

linear Fredholm integral equation of the second kind that the exact solution is $y(x)=1+\frac{10}{9} x^{2}$.

The mean errors of Bernstein collocation method obtained at the uniform and nonuniform collocation points with increasing $n$ are given in Table 1. The Table shows that the numerical results obtained at the nonuniform collocation points are better than the results obtained at the uniform collocation points. Mean errors obtained for $n=4$ by using the numerical method [8] based on the Bernstein basis polynomials is nearby $10^{-13}$, whereas mean errors of the presented method is nearby $10^{-17}$ for $n=2$. Therefore, we can say that our method is effective even for smaller value $n$.

Example 2. Consider the

$$
y(x)=\cos (x)-e^{x} \sin (x)+\int_{0}^{x} e^{x} y(t) d t ; 0 \leq x \leq 1
$$

linear Volterra integral equation of the second kind that analytic solution is $y(x)=\cos x$.

In Table 2, the root of the mean square errors of collocation method based on the generalized Bernstein polynomials at the collocation points $x_{s}=\left(1-\cos \left(\frac{\pi s}{n}\right)\right) / 2$ for $n \geq 2$ are compared with the Bernstein approach [7]. It is seen from the Table, the numerical results of proposed method converge more rapidly than the other method for $n \geq 4$. 
Table 2: Comparison the $E_{\text {root }}$ errors for Example 2.

\begin{tabular}{|c|c|c|}
\hline$n$ & Presented method & Bernstein approach [7] \\
\hline 2 & $4.7 e-003$ & $2.0 e-003$ \\
\hline 3 & $3.1 e-004$ & $2.3 e-004$ \\
\hline 4 & $4.7 e-006$ & $6.2 e-006$ \\
\hline 5 & $1.8 e-007$ & $5.4 e-007$ \\
\hline 6 & $2.1 e-009$ & $1.1 e-008$ \\
\hline 7 & $1.0 e-010$ & $1.1 e-009$ \\
\hline 8 & $1.3 e-012$ & $3.4 e-010$ \\
\hline 9 & $5.2 e-014$ & $3.3 e-010$ \\
\hline 10 & $6.6 e-016$ & $3.4 e-010$ \\
\hline
\end{tabular}

Table 3: Mean errors of Example 3.

\begin{tabular}{|c|l|l|c|c|l|}
\hline$n$ & $x_{s}=\frac{s}{n}$ & $x_{s}=\frac{1-\cos \left(\frac{\pi s}{n}\right)}{2}$ & $n$ & $x_{s}=\frac{s}{n}$ & $x_{s}=\frac{1-\cos \left(\frac{n s}{n}\right)}{2}$ \\
\hline 2 & $6.0 e-004$ & $6.0 e-004$ & 12 & $1.1 e-016$ & $1.6 e-016$ \\
\hline 3 & $5.8 e-005$ & $2.5 e-005$ & 17 & $3.4 e-016$ & $1.9 e-016$ \\
\hline 4 & $1.2 e-006$ & $4.7 e-007$ & 18 & $8.8 e-015$ & $1.9 e-016$ \\
\hline 5 & $1.1 e-007$ & $1.2 e-008$ & 19 & $3.0 e-015$ & $3.3 e-016$ \\
\hline 6 & $2.0 e-009$ & $1.3 e-010$ & 20 & $1.6 e-014$ & $2.5 e-016$ \\
\hline 7 & $1.5 e-010$ & $3.9 e-012$ & 21 & $1.2 e-013$ & $1.8 e-016$ \\
\hline 8 & $2.2 e-012$ & $3.8 e-014$ & 22 & $1.9 e-013$ & $3.0 e-016$ \\
\hline 9 & $1.4 e-013$ & $1.4 e-015$ & 23 & $9.0 e-015$ & $2.2 e-016$ \\
\hline 10 & $1.6 e-015$ & $1.6 e-016$ & 24 & $6.4 e-013$ & $2.0 e-016$ \\
\hline 11 & $1.7 e-016$ & $2.3 e-016$ & 25 & $5.9 e-014$ & $2.4 e-016$ \\
\hline
\end{tabular}

Table 4: Comparison of the $E_{r e l}$ errors for Example 3.

\begin{tabular}{|c|c|c|c|c|}
\hline & \multicolumn{2}{|c|}{$n=5$} & \multicolumn{2}{c|}{$n=7$} \\
\hline$x$ & Presented method & Galerkin method [11] & Presented method & Galerkin method [11] \\
\hline 0.1 & $1.5 e-006$ & $1.9 e-005$ & $1.4 e-009$ & $1.3 e-005$ \\
\hline 0.2 & $3.4 e-007$ & $7.2 e-006$ & $1.2 e-009$ & $3.8 e-006$ \\
\hline 0.3 & $8.6 e-007$ & $1.0 e-005$ & $3.4 e-010$ & $6.1 e-006$ \\
\hline 0.4 & $3.4 e-007$ & $1.1 e-005$ & $3.1 e-010$ & $3.5 e-006$ \\
\hline 0.5 & $2.1 e-008$ & $4.8 e-007$ & $6.4 e-010$ & $7.6 e-006$ \\
\hline 0.6 & $3.4 e-007$ & $9.2 e-006$ & $3.4 e-010$ & $1.4 e-006$ \\
\hline 0.7 & $7.1 e-007$ & $7.8 e-006$ & $3.7 e-010$ & $5.9 e-006$ \\
\hline 0.8 & $3.4 e-007$ & $3.6 e-006$ & $8.8 e-010$ & $3.3 e-006$ \\
\hline 0.9 & $6.0 e-007$ & $9.3 e-006$ & $4.5 e-010$ & $5.5 e-006$ \\
\hline 1 & $3.4 e-007$ & $2.1 e-005$ & $4.5 e-010$ & $1.5 e-005$ \\
\hline
\end{tabular}

Example 3. Consider the linear Fredholm integral equation of the second kind:

$$
y(x)=e^{x}+2 \int_{0}^{1} e^{x+t} y(t) d t ; 0 \leq x \leq 1 .
$$

Exact solution of the above equation is $y(x)=\frac{e^{x}}{2-e^{2}}$.

Mean errors obtained by using the presented method are given in Table 3. When the collocation points are not equally spaced, Table 3 shows that results obtained on these points are much better than the other results. The absolute relative 
Table 5: Mean errors of Example 4.

\begin{tabular}{|l|l|l|c|c|l|}
\hline$n$ & \multicolumn{1}{|c|}{$x_{s}=\frac{s}{n}$} & $x_{s}=\frac{1-\cos \left(\frac{\pi s}{n}\right)}{2}$ & $n$ & $x_{s}=\frac{s}{n}$ & $x_{s}=\frac{1-\cos \left(\frac{\pi s}{n}\right)}{2}$ \\
\hline 2 & $9.8 e-018$ & $9.3 e-018$ & 10 & $3.5 e-017$ & $8.1 e-017$ \\
\hline 3 & $2.3 e-017$ & $4.3 e-017$ & 14 & $7.6 e-017$ & $7.6 e-017$ \\
\hline 4 & 0 & $7.4 e-018$ & 15 & $7.7 e-017$ & $6.7 e-017$ \\
\hline 5 & $3.7 e-017$ & $1.1 e-016$ & 16 & $4.7 e-016$ & $1.1 e-016$ \\
\hline 6 & $8.2 e-017$ & $1.9 e-017$ & 18 & $5.5 e-016$ & $5.4 e-017$ \\
\hline 7 & $1.3 e-016$ & $1.8 e-016$ & 20 & $5.0 e-015$ & $9.3 e-017$ \\
\hline 8 & $2.6 e-017$ & $6.2 e-017$ & 25 & $4.8 e-014$ & $7.6 e-017$ \\
\hline 9 & $5.9 e-017$ & $7.0 e-017$ & 30 & $2.0 e-012$ & $7.3 e-017$ \\
\hline
\end{tabular}

Table 6: Comparison of the $E_{\max }$ errors for Example 4.

\begin{tabular}{|c|c|c|c|c|}
\hline$n$ & Presented method & Collocation method [3] & Fixed point method [3] & Taylor expansion method [4] \\
\hline 14 & $3.3 e-016$ & - & - & $2.0 e-015$ \\
\hline 16 & $6.7 e-016$ & $7.8 e-005$ & $3.8 e-004$ & - \\
\hline 32 & $2.5 e-012$ & $4.7 e-005$ & $9.5 e-005$ & - \\
\hline
\end{tabular}

errors are compared with the Galerkin method [11] based on the Bernstein basis polynomials in Table 4 . It shows that the results obtained on the collocation points $x_{s}=s / n$ are better than the results given by the other method.

Example 4. Consider the following linear Fredholm-Volterra integral equation of the third kind:

$$
3 y(x)=3 x^{2}-\sin x\left(x^{2} \sin x+2 x \cos x-2 \sin x-\sin 1+2 \cos 1\right)+\int_{0}^{1} \sin x \cos t y(t) d t+\int_{0}^{x} \sin x \cos t y(t) d t
$$

Analytic solution of the above equation is $y(x)=x^{2}$.

The mean errors of the presented method with increasing $n$ are given in Table 5. From the Table, we can see that the numerical results obtained at the not equally spaced points are better than the numerical results obtained at the equally spaced points.

In Figure 1, the absolute error results obtained at the collocation points $x_{s}=s / n$ by applying derivated Bernstein collocation method are presented for different values $n$. The figures show that the numerical results converge more rapidly for increasing values $n$.

The maximum errors obtained with Bernstein collocation method at the number $n+1$ collocation points $x_{s}=\left(1-\cos \left(\frac{\pi s}{n}\right)\right) / 2$ are compared with the maximum errors presented for number $n$ by Taylor expansion method [4], collocation method and fixed point method [3] in Table 6. It shows that the presented method is more effective than the other methods. Besides, the results of proposed method obtained without iteration are better than the results of collocation and fixed point methods [3] obtained with 8 th iteration.

\section{Conclusions}

In this work, a collocation method based on the generalized Bernstein polynomials has been introduced for the numerical solution of linear FVIEs directly. This method is valid for the integral equations whoose solution is continuous on the interval $[a, b]$. The error bounds and convergence of the Bernstein collocation method have been presented. Some numerical examples have been given to show the applicability and accuracy of the proposed method. The collocation 


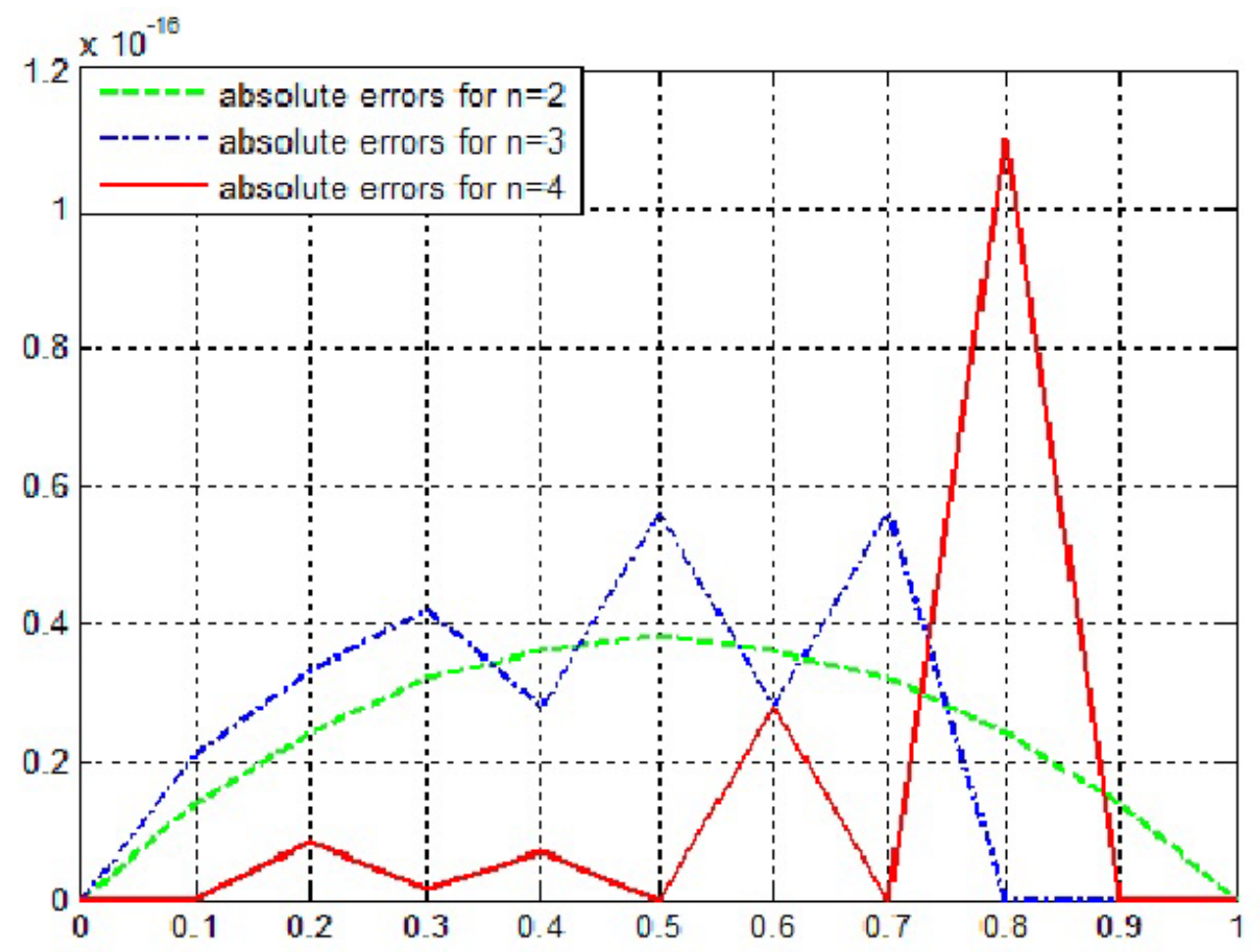

Fig. 1: The results of $\left|e_{n}(x)\right|$ absolute errors for $n=2,3,4$.

method has much better numerical results obtained at nonuniform points than the uniform points for increasing values $n$. It is also more effective method than the other methods given in the examples (1-4). Consequently, all these positive reasons are encouraged that the proposed method can be applied to the other linear and nonlinear equations.

\section{References}

[1] A. Akyüz-Daşcığlu, N. Isler Acar, Bernstein Collocation Method for Solving Linear Differential Equations, GU. J. Sci. 26 (2013) 527-534.

[2] S. Bhattacharya, B.N. Mandal, Use of Bernstein Polynomials in Numerical Solutions of Volterra Integral Equations, Appl. Math. Sci. 2 (2008) 1773-1787.

[3] F. Caliò, F. Munoz, E. Marchetti, Direct and iterative methods for the numerical solution of mixed integral equations, Appl. Math. Comput. 216 (2010) 3739-3746.

[4] Z. Chen, W. Jiang, An approximate solution for a mixed linear Volterra-Fredholm integral equation, Appl. Math. Letters 25 (2012) 1131-1134.

[5] R.T. Farouki, V.T. Rajan, Algorithms for Polynomials in Bernstein Form, Comput. Aided Geom. D. 5 (1988) 1-26.

[6] G.G. Lorentz, Bernstein polynomials, Chelsea Publishing, New York, 1986.

[7] K. Maleknejad, E. Hashemizadeh, R. Ezzati, A new approach to the numerical solution of Volterra integral equations by using Bernstein approximation, Commun. Nonlinear. Sci. Numer. Simulat. 16 (2011) 647-655.

[8] B.N. Mandal, S. Bhattacharya, Numerical Solution of some classes of integral equations using Bernstein polynomials, Appl. Math. Comput. 190 (2007) 1707-1716.

[9] M.G. Phillips, Interpolation and Approximation by Polynomials, Springer-Verlag, New York, 2003. 
[10] T.J. Rivlin, An intoduction to the approximation of functions, Dover Publications, 1969.

[11] A. Shirin, M.S. Islam, Numerical Solutions of Fredholm Integral Equations Using Bernstein Polynomials, J. Sci. Res. 2 (2) (2010) 264-272.

[12] V.K. Singh, R.K. Pandey, O.P. Singh, New stable numerical solutions of singular integral equations of Abel type by using normalized Bernstein polynomials, Appl. Math. Sci. 3 (5) (2009) 241-255. 